\title{
Successful simultaneous transcatheter treatment for secundum atrial septal defect with valvular pulmonary stenosis in children under transthoracic echocardiography guidance
}

\section{Xuning Lu}

Dalian Medical College: Dalian Medical University

Ping Wen ( $\square$ wen_dalianeryi@126.com )

Dalian Medical College: Dalian Medical University https://orcid.org/0000-0003-3450-8120

\section{Yuhang Liu}

Dalian Childrens Hospital of Dalian Medical University

Quanwei Zhu

Dalian Childrens Hospital of Dalian Medical University

\section{Research article}

Keywords: Transcatheter treatment, Atrial septal defect, Pulmonary stenosis, Transthoracic echocardiography, Children

Posted Date: June 15th, 2021

DOI: https://doi.org/10.21203/rs.3.rs-622116/v1

License: (c) (i) This work is licensed under a Creative Commons Attribution 4.0 International License.

Read Full License 


\section{Abstract}

BACKGROUND Transcatheter device closure of secundum atrial septal defect (ASD) with valvular pulmonary stenosis (PS) under fluoroscopy and/or transesophageal echocardiography (TEE) guidance is a mature technology. However, little study has focused on whether the technology can be guided totally by transthoracic echocardiography (TTE), even in children.

METHODS Thirteen children with ASD combined with PS underwent transcatheter device closure totally guided by TTE at our cardiac center from March 2016 to August 2 019. Percutaneous transcatheter pulmonary valvuloplasty was performed first and then transcatheter closure of the ASD uneventfully.

RESULTS All cases were successfully treated with transcatheter balloon pulmonary valvuloplasty and closure of ASD respectively via femoral vein approach solely under TTE guidance. The mean defect size was $8.1 \pm 1.4 \mathrm{~mm}$ (range: $5.5-10 \mathrm{~mm}$ ), the preoperative mean pressure gradient across pulmonary valve was $61.2 \pm 5.5 \mathrm{mmHg}$ (range: $51-71 \mathrm{mmHg}$ ). The mean device size used was $11.1 \pm 1.9 \mathrm{~mm}$ (range: $8-14$ $\mathrm{mm}$ ), the mean procedure time was $55.1 \pm 8.0 \mathrm{~min}$ (range: $45-71 \mathrm{~min}$ ). There were no serious cardiovascular related complications. During the follow-up period (10 mon to $47 \mathrm{mon}$ ), no arrhythmias device, thrombosis, residual fistulas or device frame fractures were detected. PS gradient had significant difference before and after procedure during the follow up $(t=28.9, P=0.000)$.

CONCLUSION Simultaneous transcatheter treatment for ASD complicated by PS in children under TTE guidance is an safe and effective therapeutic option.

\section{Background}

ASD combined with PS is a relatively uncommon congenital heart malformation, and some patients are diagnosed in children. Early intervention is sometimes required to block cardiac dysfunction. Traditional treatment includes surgical repair and transcatheter device closure. Surgical repair needs cardiopulmonary bypass, with sternotomy, high risk, many complications and slow recovery [1] Transcatheter device closure need to be exposed to radiation and contrast agent, which is limited in children [2]. To overcome these defects, we proposed a method in children in which the transcatheter technique was under TTE guidance. Only few previous reports described the successful simultaneous percutaneous transcatheter closure of ASD with PS in children. This article describes the details and outcomes of children who underwent successful simultaneous percutaneous transcatheter closure of ASD with PS by TTE guidence.

\section{Materials And Methods}

The research was approved by the Ethics Committee of Dalian Medical University, China and observed the tenets of the Declaration of Helsinki, and signed informed consent was obtained from the parents before the procedures. All patients were selected in cardiac center of dalian children's hospital affiliate to Dalian Medical University during March 2016 to August 2 019. There were 5 boys and 8 girls, the mean 
age of patients at procedure was $4.4 \pm 1.2$ years (range: 3 to 6.7 years), mean weight was $13.9 \pm 2.7 \mathrm{~kg}$ (range: 11 to $19 \mathrm{~kg}$ ). All patients were diagnosed with secundum ASD and PS by TTE, with right atrial and ventricular dilatation, with the symptoms of delayed growth, and with recurrent respiratory infections. The mean defect size was $8.1 \pm 1.4 \mathrm{~mm}$ (range: $5.5-10 \mathrm{~mm}$ ), the preoperative mean pressure gradient across pulmonary valve was $61.2 \pm 5.5 \mathrm{mmHg}$ (range: $51-71 \mathrm{mmHg}$ ). The inclusion and exclusion criteria were the same as the routine criteria used for transcatheter device closure of ASD and transcatheter pulmonary valvuloplasty.

All of the patients underwent anesthesia with midazolam and ketamine in catheterization room. The TTE was performed at the xiphoid, apex, and parasternal side to guide the entire procedure. During the process, patients were injected with 50 units $/ \mathrm{kg}$ of heparin, and the activated clotting time was monitored and maintained at a time above 200 seconds. At first, transcatheter pulmonary valvuloplasty was performed. A sheath was punctured into the right femoral vein. Next, we inserted with a multifunctional catheter and a guidewire into the venous sheath and advanced through the inferior vena cava to the right atrium. Under the guidance of real-time TTE, the multifunctional catheter and a guidewire were passed through tricuspid valve and pulmonary valve to the main pulmonary artery (Fig 1A). We choosed a Cristal balloon ( Balt Extrusion, 10 rue de la Croix-Vigneron, MONTMORENCY, FRANCE) with a diameter 1.2-1.4 times bigger than the pulmonary valve annulus (Fig 1B). The pressure gradient decreased from $61.2 \pm 5.5$ to $21.4 \pm 1.9 \mathrm{mmHg}$ after balloon dilation.

Then, transcatheter closure of ASD was performed. The size of the occluder (manufactured by Shan Dong Visee Medical Apparatus Co. Ltd. of China) was selected 2-4 mm more than echocardiographic ASD size. The transport sheath was advanced through the ASD into the left atrium (Fig 2A) under TTE guidance, then, the left and right atrial discs of appropriate occluder were released by pushing the rod, in turn, and the occluder was successfully implanted (Fig 2B). All patients received aspirin for 6 months as antithrombotic therapy after procedure.

\section{Results}

In this study, all patients were successfully treated with transcatheter balloon pulmonary valvuloplasty and closure of ASD respectively via femoral vein approach solely under TTE guidance (clinical data are shown in Table 1). The mean occluder size was $11.1 \pm 1.9 \mathrm{~mm}$ (range: 8-14 mm), the mean procedure time was $55.1 \pm 8.0 \mathrm{~min}$ (range: $45-71 \mathrm{~min}$ ) and the mean length of hospital stay was $4.8 \pm 0.9$ day (range: 4-7 day). The pressure gradient of PS had significant difference before and after procedure during the follow up $(t=28.9, P=0.000)$. 
Table 1

Clinical data of patients in this study

\begin{tabular}{|ll|}
\hline Item & \\
\hline No. of patients & 13 \\
\hline Sex (F/M) & $8 / 5$ \\
\hline Age (yr) & $4.4 \pm 1.2$ \\
\hline Body weight (kg) & $13.9 \pm 2.7$ \\
\hline ASD size (mm) & $8.1 \pm 1.4$ \\
\hline Device size (mm) & $11.1 \pm 1.9$ \\
\hline Preoperative PS gradient(mmHg) & $61.2 \pm 5.5$ \\
\hline Postoperative PS gradient(mmHg) & $21.4 \pm 1.9$ \\
\hline Procedure time (min) & $55.1 \pm 8.0$ \\
\hline Hospital stay (day) & $4.8 \pm 0.9$ \\
\hline Follow-up (mon) & $24.0 \pm 10.8$ \\
\hline Successful rate & $100 \%$ \\
\hline ASD: Secundum atrial septal defect; PS: Valvular pulmonary stenosis \\
\hline
\end{tabular}

No deaths or serious cardiovascular related complications occurred during hospitalization. There were no fatal complications associated with occluder dislodgement, residual shunt, complete atrioventricular block, or thrombosis-related disease. A small amount of pulmonary valve regurgitation occurred after pulmonary valve dilation in 3 children.

During the follow-up period (( 10 mon to $47 \mathrm{mon})$, the patients were regularly assessed by physical examination, TTE, and electrocardiogram, no arrhythmias device, thrombosis, residual fistulas or device frame fractures were detected, and pulmonary valve regurgitation did not worsen.

\section{Discussion}

ASD is one of the most common congenital acyanotic heart diseases [3], however, the association with PS is a relatively uncommon [4]. When this condition is present simultaneously, significant left-to-right shunting is often prevented by the outflow obstruction, which can shelter the pulmonary vascular bed without premature damage until adulthood. But, persistence of this complication has harmful effects on right ventricular systolic function [5], in addition, it can be indicated by symptoms, such as recurrent respiratory infections and delayed growth [6-8]. This fact indicates that total correction is necessary. 
For most patients with ASD and PS, surgical repair and transcatheter device closure have been demonstrated satisfactory in terms of short- and long term results. Traditional surgery requires thoracotomy and cardiopulmonary bypass. Cardiopulmonary bypass can bring more complications and more traumatic, include systemic inflammatory response and multiple organ dysfunction. In addition, huge surgical scars are unacceptable for many parents [9-10]. In recent years, with the improvement of various new occluders and cardiovascular-interventional technology, transcatheter closure of ASD and pulmonary valve balloon dilatation have been widely used worldwide, and has become the first choice to deal with these diseases. Transcatheter therapy has many attractive advantages, including poor pain, less trauma, and shorter hospital stay [11-12]. However, its biggest drawback is that both doctors and patients are exposed to $\mathrm{x}$-ray radiation, which can cause radiation-related damage. In order to avoid X-ray radiation, ultrasound is used instead of X-rays in interventional treatment [13-14].

Ultrasound mainly includes transesophageal echocardiograph (TEE) and TTE. Although it is well known that the image of TEE is more accurate than TTE, many experts have shown that TTE can be used as the only guiding tool to complete transcatheter balloon pulmonary valvuloplasty and closure of ASD, and in some cases can completely replace TEE [15-16]. Pan et al [17] summarized their experience that transcatheter closure of ASD used solely TTE guidance. Galal et al [18] reported that TTE was used as the only imaging tool to complete PS balloon dilation. Their method avoided fluoroscopy and/or tracheal intubation, and with satisfactory results. Therefore, TTE was chosen as the only imaging too to complete transcatheter balloon pulmonary valvuloplasty and closure of ASD.

Both malformations were corrected in one intervention, thereby avoiding the need for a second interventional procedure [19]. It is important to choose a correct and reasonable sequence of treatments to ensure the safety of transcatheter interventional therapy. The first procedure that should be performed is controversial. Some reports recommend percutaneous pulmonary valvuloplasty before transcatheter closure of ASD, which would restrict the possibility of dislocation of the ASD device [20-21], in addition, ASD can be used as a shunt during percutaneous pulmonary valvuloplasty. We chose to performe pulmonary valvuloplasty first and then closure of the ASD. We have accumulated some important experiences during theprocedure, which would help improve TTE guidance on simultaneous transcatheter correction. First, when the tip of the catheter entered the left and right atria, it was usually unclearly displayed in the apical four-chamber or parasternal short-axis view by TTE. Therefore, we often rotated the catheter or acoustic contrast by injected a small amount of water from the catheter in order to show the catheter tip clearly. Second, proper pre-expanded balloon is necessary to overcome the difficulty of showing the position of the balloon in the pulmonary valve in parasternal short-axis view by TTE. Preexpanded balloon should be careful and as little force as possible to effectively prevent obstruction of right ventricular outflow tract.

\section{Limitations}

The duration of follow-up was short in this research, which must be improved in the following studies. In addition, this is a single-centre, retrospective study. The patients of study was low in the current research. 
Therefore, the multiple-centre study with a larger sample size and a long-term follow-up is mandatory in the future research to evaluate the related complications and benefits of this technique more exactly.

\section{Conclusion}

Simultaneous transcatheter treatment for ASD complicated by PS in children under TTE guidance is an safe and effective therapeutic option. This method avoids surgical scars, cardiopulmonary bypass, fluoroscopy, and endotracheal intubation, and associated with a satisfactory success rate.

\section{Abbreviations}

ASD secundum atrial septal defect

PS valvular pulmonary stenosis

TTE transthoracic echocardiography

TEE transesophageal echocardiograph

\section{Declarations}

\section{Ethics approval and consent to participate}

The study was approved by the Ethics Committee of Dalian Children's Hospital affiliated with Dalian Medical University.

\section{Consent for publication}

Not applicaple.

\section{Availability of data and material}

The datasets generated and analysed during the current study are available upon reasonable request from the corresponding authors.

\section{Competing interests}

The authors have no conflicts of interest to declare.

\section{Funding}

This work was no supported.

\section{Authors' contributions}


Conceived and designed the experiments: Xu-Ning Lu

Performed the experiments: Xu-Ning Lu, Ping Wen, Yu-Hang Liu

Analyzed the data: Xu-Ning Lu, Ping Wen, Yu-Hang Liu

Contributed reagents/materials/analysis tools: Xu-Ning Lu

\section{Acknowledgements}

The authors acknowledge all people who helped us in preparation of this study. We have to appreciate officials, head nurse, and nurses of echocardiography room and operating room of dalian children's hospital affiliate to Dalian Medical University.

\section{References}

1. Hong Luo, Jing Wang, Chenhui Qiao, Xin Zhang, Weihua Zhang, Laichun Song. Evaluation of different minimally invasive techniques in the surgical treatment of atrial septal defect. J Thorac Cardiovasc Surg 2014; 148: 188-193.

2. Boudoulas KD, Marmagkiolis K, Boudoulas H. Atrial Septal Defect Sizing and Transcatheter Closure. Cardiology 2019; 142:105-108.

3. Tal Geva, Jose D Martins, Rachel M Wald. Atrial septal defects. Lancet 2014; 383(9932):1921-32.

4. Lara DA1, Lopez KN. Public health research in congenital heart disease. Congenit Heart Dis 2014; 9: 549-58.

5. Xu-Dong Xu, Su-Xuan Liu, Xian-Xian Zhao, Yong-Wen Qin. Comparison of medium-term results of transcatheter correction versus surgical treatment for secundum type atrial septal defect combined with pulmonary valve stenosis. Int Heart J 2014; 4: 326-330.

6. Geggel RL. Clinical detection of hemodynamically significant isolated secundum atrial septal defect. J Pediatr 2017; 190: 261-4.

7. John J Parent, Michael M Ross, Edgard A Bendaly, John P Breinholt. Results of pulmonary balloon valvuloplasty persist and improve at late follow-up in isolated pulmonary valve stenosis. Cardiol Young $2017 ; 27: 1566-1570$

8. Astrid Lammers, Alfred Hager, Andreas Eicken, Rüdiger Lange, Michael Hauser, John Hess. Need for closure of secundum atrial septal defect in infancy. J Thorac Cardiovasc Surg 2005; 129(6): 1353-7. 
9. Hirata Y. Cardiopulmonary bypass for pediatric cardiac surgery. Gen Thorac Cardiovasc Surg 2018; 66: 65-70.

10. Michael G Earing, Heidi M Connolly, Joseph A Dearani, Naser M Ammash, Martha Grogan, Carole A Warnes. Long-term follow-up of patients after surgical treatment for isolated pulmonary valve stenosis. Mayo Clin Proc 2005; 80: 871-6.

11. Charan Lanjewar, Milind Phadke, Arvind Singh, Girish Sabnis, Mahesh Jare, Prafulla Kerkar. Percutaneous balloon valvuloplasty with Inoue balloon catheter technique for pulmonary valve stenosis in adolescents and adults. Indian Heart J 2017; 69:176-181.

12. Yang MC, Wu JR. Recent review of transcatheter closure of atrial septal defect. Kaohsiung J Med Sci 2018; 34(7): 363-369 .

13. Weize Xu, Jianhua Li, Jingjing Ye, Jin Yu, Jiangen Yu, Zewei Zhang. Transesophageal echocardiography and fluoroscopy for percutaneous closure of atrial septal defects: A comparative study. Medicine (Baltimore) 2018; 97: e12891.

14. Tuğba Kemaloğlu Öz, Fatma Özpamuk Karadeniz, Şükrü Akyüz, Şennur Ünal Dayı, Aycan Esen Zencirci, Işıl Atasoy, Altuğ Ösken, Mehmet Eren. The advantages of live/real time three-dimensional transesophageal echocardiography during assessments of pulmonary stenosis. Int $\mathrm{J}$ Cardiovasc Imaging $2016 ; 32: 573-82$.

15. Kobayashi T. Monitoring tool of transcatheter atrial septal defect: transesophageal echocardiography or transthoracic echocardiography? J Cardiol 2015; 65:1

16. Qiang Chen, Hua Cao, Gui-Can Zhang, Liang-Wan Chen, Fan Xu. Successful totally transthoracic echocardiography guided transcatheter device closure of atrial septal defect in pregnant women. World $\mathrm{J}$ Clin Cases 2019; 7:734-741

17. Xiang-Bin Pan, Wen-Bin Ou-Yang, Kun-Jing Pang, Feng-Wen Zhang, Shou-Zheng Wang, Yao Liu, DaWei Zhang, Gai-Li Guo, Peng-Sheng Tian, Sheng-Shou Hu. Percutaneous Closure of Atrial Septal Defects Under Transthoracic Echocardiography Guidance Without Fluoroscopy or Intubation in Children. J Interv Cardiol 2015; 28: 390-395 .

18. Galal MO, Jadoon S, Momenah TS. Pulmonary Valvuloplasty in a Pregnant Woman Using Sole Transthoracic Echo Guidance: Technical Considerations. Canadian Journal of Cardiology 2015; 31: 103.e5-103.e7.

19. Saritas Turkay, Erdem Abdullah, Akdeniz Celal, Zeybek Cenap, Erol Nurdan, Demir Fadli, Demir Halil, Aydemir Numan Ali, Celebi Ahmet. Multiple transcatheter interventions in the same session in congenital cardiopathies. J Cardiovasc Dis Res 2010; 4: 181-190. 
20. Asada D, Tomita H, Fujii T. Successful simultaneous transcatheter treatment for a secundum atrial septal defect complicated by valvular pulmonary stenosis in an infant. Cardiol Young 2018; 28:11621164.

21. Sebastian Smerdziński, Jacek Białkowski, Małgorzata Szkutnik, Mateusz Knop, Michał Gałeczka, Roland Fiszer. Long-term effects of combined percutaneous atrial septal defect occlusion and pulmonary valvuloplasty in children $<2.5$ years old. Kardiol Pol 2019; 77: 53-55.

\section{Figures}
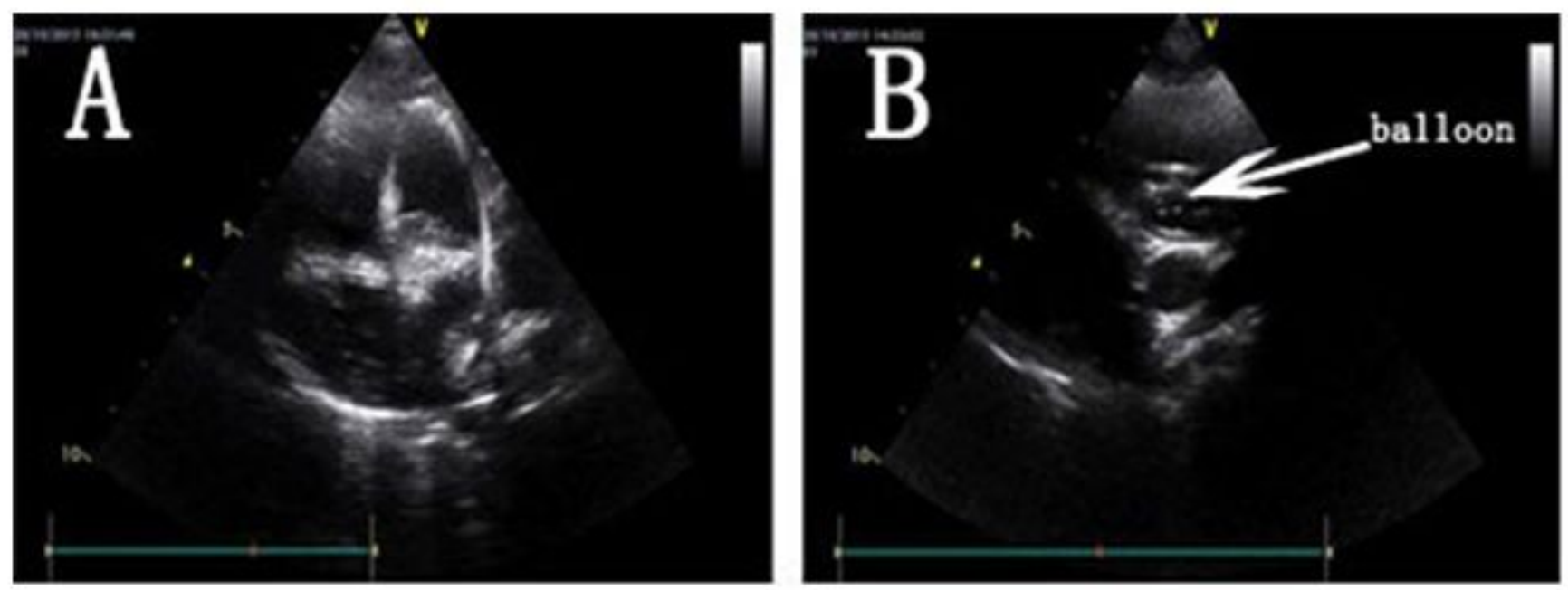

\section{Figure 1}

Transthoracic echocardiography showing the process of transcatheter pulmonary valvuloplasty. A: A multifunctional catheter was crossed the pulmonary valve and reaching the right pulmonary artery; $\mathrm{B}$ : The balloon was dilated at the position of the pulmonary valve
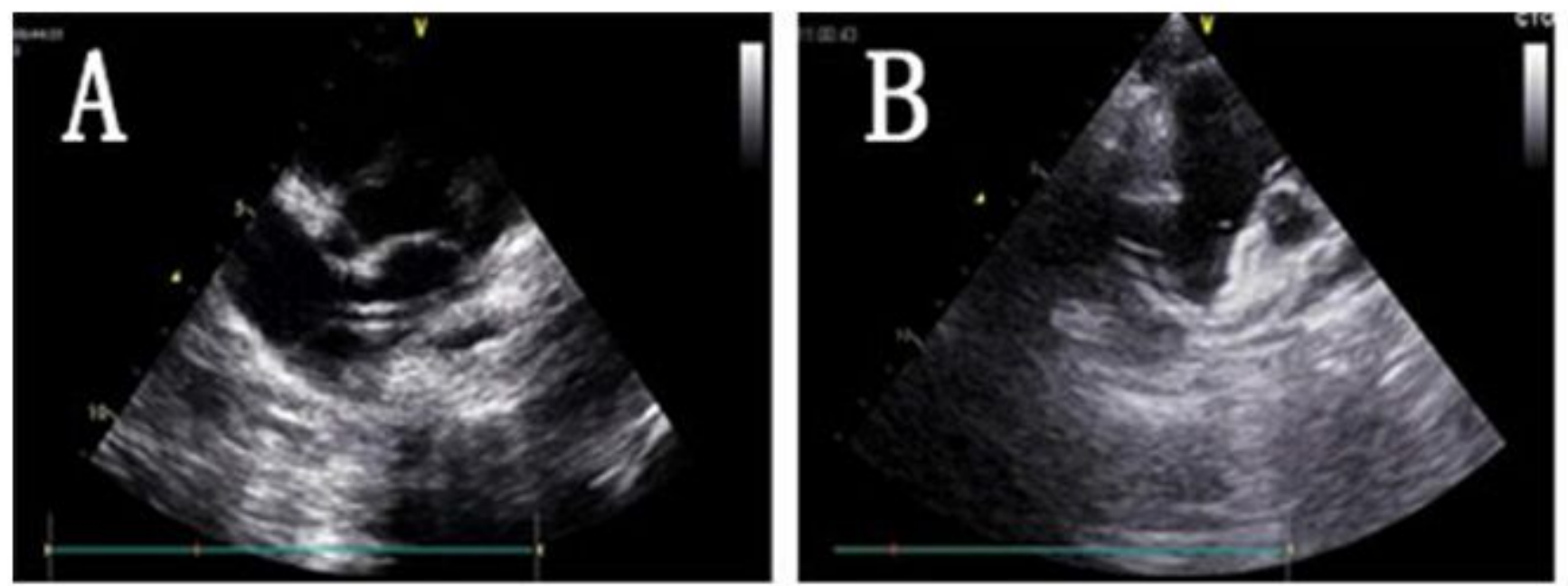

Figure 2 
Transthoracic echocardiography showing the process of transcatheter device closure of atrial septal defect. A: A delivery sheath was inserted into the left atrium under the guidance of echocardiography ; B: The occluder was released. 